\title{
An Integral Expression for the Electrical Potential Distribution for Charged Surfaces in Electrolyte Solutions
}

\author{
Jyh-PING Hsu ${ }^{1}$ AND Ming-Tsan TsEng \\ Department of Chemical Engineering, National Taiwan University, Taipei, Taiwan 10617, Republic of China
}

Received September 26, 1996; accepted December 13, 1996

\begin{abstract}
An analytical procedure is suggested for the resolution of the linearized Poisson- Boltzmann equation governing the electrical potential distribution of a charged surface in an electrolyte solution under the Debye- H uckel condition. An Integral expression based on the $\mathrm{G}$ reen function of the linearized Poisson - B oltzmann equation is derived. The electrical potential distributions for the following cases are solved to illustrate the present method: an arbitraryshaped thin surface, a porous (ion-penetrable) sphere bearing fixed charges, a rigid, planar surface covered by a porous membrane bearing fixed charges, and a rigid, nonuniformly charged planar surface. $\odot 1997$ Academic Press

Key Words: Poisson - Boltzmann equation, linearized; H elmholtz equation; electrical potential distribution; Green function representation; general expression; rigid charged surface; planar; nonuniform surface condition; rigid charged surface; thin, arbitrary shape; porous object, ion-penetrable, planar, or spherical.
\end{abstract}

\section{INTRODUCTION}

The electrical potential distribution for a charged surface in a vacuum is governed by the Poisson equation. It can be solved by considering an equivalent integral equation containing the Green function of the Poisson equation and the charge density of the surface (1). The integral equation is based on the principle that the electrical potential of the system under consideration is the sum of the electrical potentials contributed by each individual charges on the surface. For a charged surface immersed in an electrolyte solution, the electrolyte provides an additional charge source. In this case, if the concentration of electrolyte follows the Boltzmann distribution, the electrical potential distribution of the system under consideration is governed by the PoissonBoltzmann equation (PBE) (2). For an infinite planar surface in a symmetric electrolyte solution, the solution to the PBE can be derived analytically (2). Other than this case, the PBE needs to be solved either numerically (3-5) or approximately (6-9). The difficulty of solving the PBE depends largely on the geometry of a charged surface, the associated boundary conditions, and the type of electrolyte. In general, a nonlinear equation needs to be considered. If a surface is not highly charged, or if its surface potential is sufficiently low (the Debye-Huckel condition), the corresponding PBE can be approximated by the Helmholtz equation, the solution of which may be expressed as a linear combination of harmonic functions $(10-13)$. The DebyeHuckel condition is satisfactory for a surface potential on the order of $25 \mathrm{mV}$. This may be violated for some of the charged surfaces in practice. Nevertheless, it is often adopted in the literature for a simpler treatment. Although reported results are ample in the literature, most of them are based on simple geometries such as planar (13), cylindrical (9), spherical (10-11), and spheroidal surfaces (14-15), and uniform surface conditions, for example, uniform surface potential or uniform surface charge density. Since a charged surface can take virtually any shape, and various surface conditions can be assumed in practice, a more general treatment is highly desirable.

In the present study the solution procedure for the electrical potential distribution around a charged surface in an electrolyte solution under the Debye-Huckel condition is discussed. The types of charged surfaces examined include: an arbitrary-shaped thin rigid surface, a porous (ion-penetrable) sphere bearing fixed charges, a planar surface covered by a porous membrane bearing fixed charges, and a rigid, nonuniformly charged planar surface. The mathematical treatment is based on the Green function of the linearized PBE. A similar approach was adopted by Halle (16) for the case of two infinitely thin rodlike macroions.

\section{THEORY}

Consider first a unit positive fixed point charge located at the dimensionless position $\bar{x}=\left[x_{1}, x_{2}, x_{3}\right]$ in an electrolyte solution. The dimensionless electrical potential distribution induced by this charge, $G$, is described by the Helmholtz equation

$$
\nabla_{\bar{\epsilon}}^{2} G(\bar{\epsilon}, \bar{x})=G(\bar{\epsilon}, \bar{x})-\delta(\bar{\epsilon}-\bar{x}),
$$


where $\nabla_{\bar{\epsilon}}^{2}$ is the Laplace operator based on the coordinates $\bar{\epsilon}=\left[\epsilon_{1}, \epsilon_{2}, \epsilon_{3}\right]$, and $\delta$ the delta function. The dimensionless variables are defined as

$$
\begin{aligned}
G & =e \Phi / k_{B} T \\
x_{i} & =\kappa \tilde{x_{i}}, i=1,2,3 \\
\kappa & =\left(\sum e^{2} Z_{i}^{2} C_{i, 0} / \epsilon_{r} \epsilon_{0} k_{B} T\right)^{1 / 2},
\end{aligned}
$$

where $e$ is the elementary charge, $\Phi$ the electrical potential, $\epsilon_{\mathrm{r}}$ and $\epsilon_{0}$, respectively, the relative permittivity and the permittivity of a vacuum, $Z_{i}$ the valence of ion species $i, k_{\mathrm{B}}$ the Boltzmann constant, $T$ the absolute temperature, $C_{i, 0}$ the bulk concentration of ion species $i, \kappa^{-1}$ the Debye length, and $\tilde{x}_{i}, i=1,2,3$, the position variables. The solution to Eq. [1] is

$$
G(\bar{\epsilon}, \bar{x})=\frac{\exp (-r(\bar{\epsilon}, \bar{x}))}{4 \pi r(\bar{\epsilon}, \bar{x})}
$$

where $r(\bar{\epsilon}, \bar{x})$ is the distance between an arbitrary point $\bar{\epsilon}$ and $\bar{x}$. For a distribution of fixed charges $\rho(\bar{\epsilon})$ in the electrolyte solution the electrical potential distribution, $\Psi$, is governed by

$$
\nabla_{\bar{\epsilon}}^{2} \Psi(\bar{\epsilon})=\Psi(\bar{\epsilon})-\rho(\bar{\epsilon})
$$

Define

$$
v(\bar{\epsilon})=\int_{V} G(\bar{\epsilon}, \bar{x}) \rho(\bar{x}) d \bar{x}
$$

where the right-hand side of this expression denotes the volume integral over $V$. Employing the Laplace operator on Eq. [4] yields

$$
\begin{aligned}
\nabla_{\bar{\epsilon}}^{2} v(\bar{\epsilon}) & =\int_{V} \nabla_{\bar{\epsilon}}^{2} G(\bar{\epsilon}, \bar{x}) \rho(\bar{x}) d \bar{x} \\
& =\int_{V}[G(\bar{\epsilon}, \bar{x})-\delta(\bar{\epsilon}, \bar{x})] \rho(\bar{x}) d \bar{x} \\
& =\int_{V} G(\bar{\epsilon}, \bar{x}) \rho(\bar{x}) d \bar{x}-\rho(\bar{\epsilon}) \\
& =v(\bar{\epsilon})-\rho(\bar{\epsilon}) .
\end{aligned}
$$

This implies that $v$ satisfies Eq. [3]; i.e.,

$$
\Psi(\bar{\epsilon})=\int_{V} G(\bar{\epsilon}, \bar{x}) \rho(\bar{x}) d \bar{x} .
$$

The expression was derived previously for the case the function $G$ on the right-hand side of Eq. [1] vanishes (1).
Consider next an infinitesimally thin charged surface immersed in an electrolyte solution. If the surface is not highly charged, the potential distribution satisfies

$$
\nabla_{\bar{\epsilon}}^{2} \Psi(\bar{\epsilon})=\Psi(\bar{\epsilon}), \quad \bar{\epsilon} \in E
$$

where $E$ is the domain excluding the charged surface. By referring to Fig. 1, the boundary condition associated with Eq. [7] is

$$
\left.\frac{\partial \Psi}{\partial \bar{n}}\right|_{\bar{p}^{+}}-\left.\frac{\partial \Psi}{\partial \bar{n}}\right|_{\bar{p}^{-}}=\rho(\bar{p}), \quad \bar{p} \in \Omega,
$$

where $(\partial \Psi / \partial \bar{n})_{\bar{p}}$ is the directional derivative of $\Psi$ at point $\bar{p}, \rho(\bar{p})$ the surface charge distribution at $\bar{p}, \Omega$ the domain of the charged surface, $\bar{n}$ the unit outer normal, and $\bar{p}^{+}$and $\bar{p}^{-}$the points immediately above and below $\bar{p}$, respectively. Define the function $u(\bar{\epsilon})$ as

$$
u(\bar{\epsilon})=\int_{\Omega} G(\bar{\epsilon}, \bar{x}) \rho(\bar{x}) d \Omega, \quad \bar{x} \in \Omega
$$

where the right-hand side of this expression denotes the surface integral over the charged surface. It can be shown that $u(\bar{\epsilon})$ satisfies Eq. [7], and

$$
\begin{aligned}
& \left.\frac{\partial u}{\partial \bar{n}}\right|_{\bar{p}^{+}}=\left.\int_{\Omega} \frac{\partial G(\bar{\epsilon}, \bar{x})}{\partial \bar{n}}\right|_{\bar{\epsilon}=\bar{p}} \rho(\bar{x}) d \Omega+\frac{1}{2} \rho(\bar{p}) \\
& \left.\frac{\partial u}{\partial \bar{n}}\right|_{\bar{p} \overline{-}^{-}}=\left.\int_{\Omega} \frac{\partial G(\bar{\epsilon}, \bar{x})}{\partial \bar{n}}\right|_{\bar{\epsilon}=\bar{p}} \rho(\bar{x}) d \Omega-\frac{1}{2} \rho(\bar{p}) .
\end{aligned}
$$

These expressions imply that $u$ satisfies Eq. [8], and, therefore, is the solution of Eq. [7]; i.e.,

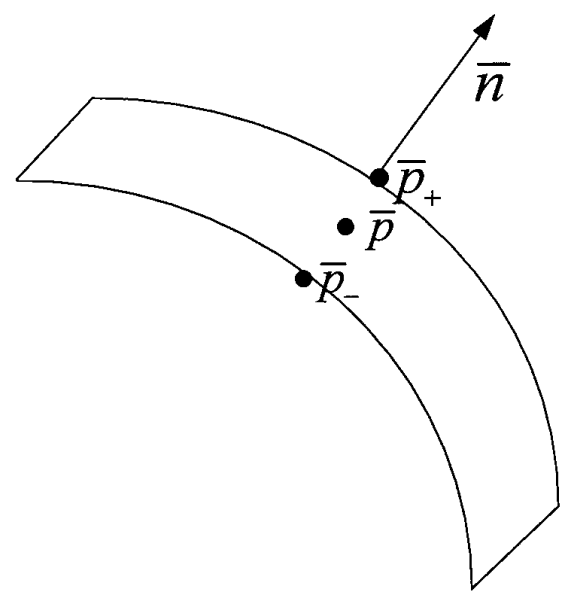

FIG. 1. A schematic representation of an arbitrary thin surface. $\bar{n}$ is the unit outer normal, $\bar{p}$ the position of an arbitrary point on the surface, $\bar{p}^{+}$ and $\bar{p}$ the points immediately above and below $\bar{p}$, respectively. 


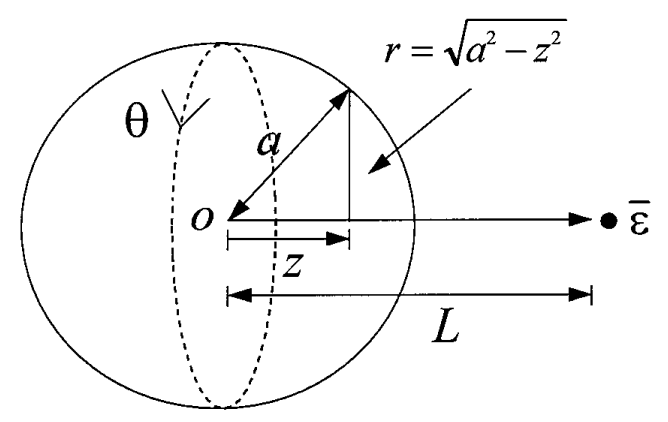

FIG. 2. The coordinates used for a porous (ion-penetrable) sphere of radius $a$. The center of the sphere is located at the origin $O \cdot \bar{\epsilon}$ denotes an arbitrary point, and $L$ is the distance between $\bar{\epsilon}$ and $O$.

$$
\Psi(\bar{\epsilon})=\int_{S} G(\bar{\epsilon}, \bar{x}) \rho(\bar{x}) d S_{\bar{x}}
$$

Note that the function $G$ defined above is the generalized Green function of the Helmholtz equation, Eq. [1].

\section{EXAMPLES}

Several examples are discussed to illustrate the applicability of the present method.

\subsection{Porous (Ion-Penetrable) Sphere Bearing Fixed Charges}

Let us consider a porous sphere bearing fixed charges with radius $a$ in an electrolyte solution. For illustration, we assume that the fixed charge distribution, $\rho(\bar{\epsilon})$, is

$$
\rho(\bar{\epsilon})=\left\{\begin{array}{ll}
\rho_{0}, & \sqrt{\epsilon_{1}^{2}+\epsilon_{2}^{2}+\epsilon_{3}^{2}} \leqslant a \\
0, & \sqrt{\epsilon_{1}^{2}+\epsilon_{2}^{2}+\epsilon_{3}^{2}}>a
\end{array} .\right.
$$

For the coordinate system shown in Fig. 2, we have, on the basis of Eq. [6],

$$
\begin{aligned}
& \Psi=\frac{1}{4 \pi} \int_{-a}^{a} \int_{0}^{\sqrt{a^{2}-z^{2}}} \int_{0}^{2 \pi} \frac{\exp \left(-\sqrt{r^{2}+(z-L)^{2}}\right)}{\sqrt{r^{2}+(z-L)^{2}}} \rho_{0} r d \theta d r d z \\
& =\frac{-\rho_{0}}{2 L}(\exp (-\alpha)(\alpha+1)) \mid \begin{array}{l}
\alpha=|a-L| \\
\alpha=|a+L|
\end{array} \\
& +\frac{\rho_{0}}{2} \int_{-a}^{a} \exp (-|z-L|) d z \\
& =\left\{\begin{array}{r}
\rho_{0} \exp (a-L)[a-1+\exp (-2 a)(a+1)] / 2 L, \\
\text { if } L>a \\
\rho_{0}(a+1) \exp (L-a)[-1+\exp (-2 L)] / 2 L+1, \\
\text { if } L<a .
\end{array}\right.
\end{aligned}
$$

The same result can be obtained by solving the PoissonBoltzmann equation for the present case

$$
\frac{d^{2} \Psi}{d p^{2}}+\frac{2 d \Psi}{p d p}=\Psi-\rho(p), \quad p \geqslant 0 .
$$

This problem was discussed by Ohshima (12), and he arrived at

$$
\Psi=\left\{\begin{array}{l}
\Psi_{0} a \exp (-(L-a)) / L, \quad L \geqslant a \\
{\left[\int_{0}^{L} r^{\prime} \rho_{0} \sinh \left(r^{\prime}-L\right) d r^{\prime}\right.} \\
\left.\quad+\sinh (L) \int_{0}^{a} r^{\prime} \rho_{0} \exp \left(-r^{\prime}\right) d r^{\prime}\right] / L, L \leqslant a,
\end{array}\right.
$$

where $r^{\prime}$ is a dummy variable and

$$
\Psi_{0}=\exp (-a) / a \int_{0}^{a} r^{\prime} \rho\left(r^{\prime}\right) \sinh \left(r^{\prime}\right) d r^{\prime}
$$

A similar problem was examined by Hsu et al. (17). Note that the present approach is applicable to an arbitrary-shaped porous object.

\subsection{Planar Surface Covered by a Membrane Bearing \\ Fixed Charges}

By referring to Fig. 3, we consider a planar rigid surface covered by an ion-penetrable membrane bearing fixed charges. Suppose that the dielectric constant of the rigid solid phase is small compared to that of the membrane phase. Let the charge density of the rigid surface and the fixed charge distribution in the membrane be $\rho_{s}(\bar{\epsilon})$ and $\rho_{V}(\bar{\epsilon})$,

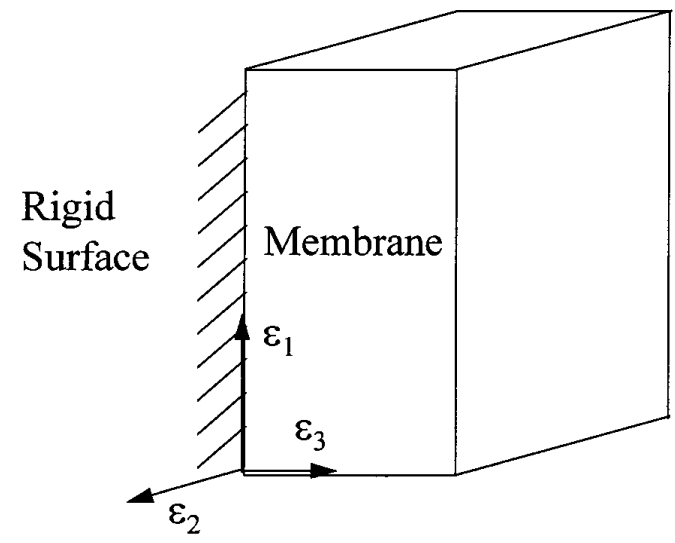

FIG. 3. A schematic representation of a rigid surface covered by an ion-penetrable charged membrane. The rigid surface-membrane interface is located at $\epsilon_{3}=0$. 
respectively. In this case, the electrical potential distribution is governed by

$$
\nabla_{\bar{\epsilon}}^{2} \Psi(\bar{\epsilon})=\Psi(\bar{\epsilon})-\rho_{V}(\bar{\epsilon}), \quad \epsilon_{3}>0
$$

The associated boundary condition is

$$
\frac{\partial \Psi}{\partial \epsilon_{3}}=-\rho_{s}(\bar{\epsilon}), \quad \epsilon_{3}=0 .
$$

The problem under consideration can be reformulated through an image method (11-12). Let us consider the problem

$$
\begin{gathered}
\nabla_{\bar{\epsilon}}^{2} \Psi(\bar{\epsilon})=\Psi(\bar{\epsilon})-\rho_{V}(\bar{\epsilon}), \quad \epsilon_{3}>0 \\
\nabla_{\bar{\epsilon}}^{2} \Psi(\bar{\epsilon})=\Psi(\bar{\epsilon})-\rho_{V}^{\prime}(\bar{\epsilon}), \quad \epsilon_{3}<0 \\
\left.\frac{\partial \Psi}{\partial \epsilon_{3}}\right|_{\epsilon_{3}=0^{+}}-\left.\frac{\partial \Psi}{\partial \epsilon_{3}}\right|_{\epsilon_{3}=0^{-}}=-2 \rho_{s}(\bar{\epsilon}), \quad \epsilon_{3}=0,
\end{gathered}
$$

where $\rho_{V}^{\prime}(\bar{\epsilon})$ is the image charge distribution satisfying

$$
\rho_{V}^{\prime}\left(\epsilon_{1}, \epsilon_{2}, \epsilon_{3}\right)=\rho_{V}\left(\epsilon_{1}, \epsilon_{2},-\epsilon_{3}\right), \quad \epsilon_{3}<0 .
$$

Equations [19]-[22] suggest that the electrical potential distribution is symmetric about $\epsilon_{3}=0$. Therefore,

$$
\left.\frac{\partial \Psi}{\partial \epsilon_{3}}\right|_{\epsilon_{3}=0^{+}}=-\left.\frac{\partial \Psi}{\partial \epsilon_{3}}\right|_{\epsilon_{3}=0^{-}}
$$

and

$$
\left.\frac{\partial \Psi}{\partial \epsilon_{3}}\right|_{\epsilon_{3}=0^{+}}=-\rho_{s}(\bar{\epsilon}) .
$$

This implies that the solution to the reformulated problem, Eqs. [19]-[24], in the region $\epsilon_{3}>0$, is the same as that of Eqs. [17] and [18]. According to Eqs. [6] and [12], the former can be expressed as

$$
\begin{aligned}
\Psi(\bar{\epsilon})= & \int_{-\infty}^{\infty} \int_{-\infty}^{\infty} G(\bar{\epsilon}, \bar{x})\left(2 \rho_{s}(\bar{x})\right) d x_{1} d x_{2} \\
& +\int_{-\infty}^{\infty} \int_{-\infty}^{\infty} \int_{-\infty}^{0-} G(\bar{\epsilon}, \bar{x}) \rho_{V}^{\prime}(\bar{x}) d x_{3} d x_{1} d x_{2} \\
& +\int_{-\infty}^{\infty} \int_{-\infty}^{\infty} \int_{0^{+}}^{\infty} G(\bar{\epsilon}, \bar{x}) \rho_{V}(\bar{x}) d x_{3} d x_{1} d x_{2} .
\end{aligned}
$$

The special case in which $\rho_{V}=\rho_{V}\left(\epsilon_{3}\right)$ and $\rho_{S}=0$ was analyzed by Ohshima (18). In this case, the first term on the right-hand side of Eq. [25] vanishes. A similar problem was examined by Hsu et al. (19).

3.2.1. Nonuniformly charged planar surface. Let us consider a planar surface with surface condition

$$
\left.\frac{\partial \Psi}{\partial \epsilon_{3}}\right|_{\epsilon_{3}=0^{+}}=-g\left(\epsilon_{1}, \epsilon_{2}\right) .
$$

Applying Eq. [25] yields

$$
\Psi(\bar{\epsilon})=\int_{-\infty}^{\infty} \int_{-\infty}^{\infty} G(\bar{\epsilon}, \bar{x})\left(2 g\left(x_{1}, x_{2}\right)\right) d x_{1} d x_{2} .
$$

As an example, let us consider the case

$$
g\left(\epsilon_{1}, \epsilon_{2}\right)=\cos \left(\epsilon_{1}\right) \cos \left(\epsilon_{2}\right)+1,-\infty<\epsilon_{1}, \epsilon_{2}<\infty .
$$

Substituting Eq. [28] into Eq. [27] gives the electrical potential distribution. In practice, the computational effort can be reduced significantly if the infinite domain in Eq. [27] is replaced by an adequately chosen finite domain $S$. Suppose that $g\left(\epsilon_{1}, \epsilon_{2}\right)$ is bounded, i.e.,

$$
\left|g\left(\epsilon_{1}, \epsilon_{2}\right)\right|<g_{\max }, \quad-\infty<\epsilon_{1}, \epsilon_{2}<\infty
$$

where $g_{\max }$ is a certain finite value. Then $S$ can be chosen as

$$
\begin{array}{r}
S=\left\{\left(\epsilon_{1}, \epsilon_{2}\right) \mid \sqrt{\left(\epsilon_{1}-x_{1}\right)^{2}+\left(\epsilon_{2}-x_{2}\right)^{2}} \leqslant R_{\max },\right. \\
\left.R_{\max }>\ln \left(4 \pi g_{\max } / \delta\right)\right\},
\end{array}
$$

where $\delta$ is a prespecified degree of accuracy.

Figure 4 shows the spatial electrical potential distribution calculated by Eqs. [27] and [30] with $\delta<0.001, g_{\max }=2$, and $R_{\max }=10.13$. In a discussion of the interaction between two nonuniformly charged surfaces, Kuin (13) assumed a periodic surface charge distribution. It is a special case of Eq. [27].

3.2.2. Thin, planar-charged surface. Let us consider a thin, planar charged surface on the $\epsilon_{1}-\epsilon_{2}$ plane with surface condition

$$
\left.\frac{\partial \Psi}{\partial \epsilon_{3}}\right|_{\epsilon_{3}=0^{+}}=-g\left(\epsilon_{1}, \epsilon_{2}\right) .
$$

Here, we assume that the dielectric constant of the solid phase is small, and that the electrical potential distribution inside is negligible. Employing Eq. [25] leads to

$$
\Psi(\bar{x})=\int_{S} G(\bar{\epsilon}, \bar{x})\left(2 g\left(\epsilon_{1}, \epsilon_{2}\right)\right) d \epsilon_{1} d \epsilon_{2} .
$$




\section{$\varepsilon_{3}=0$}

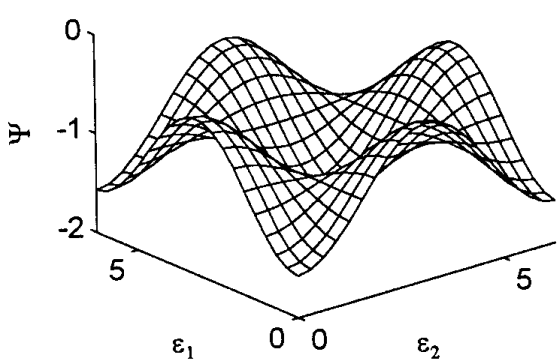

$\varepsilon_{3}=1.333$

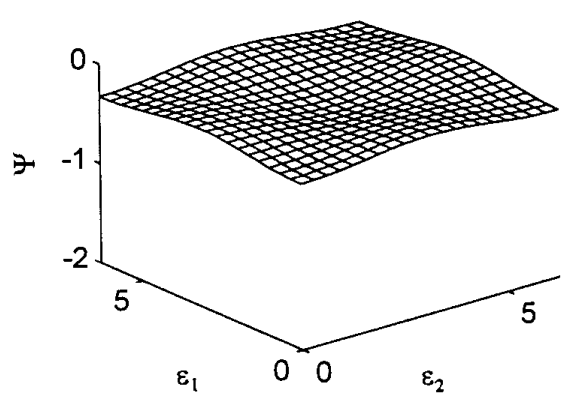

$\varepsilon_{3}=0.6667$

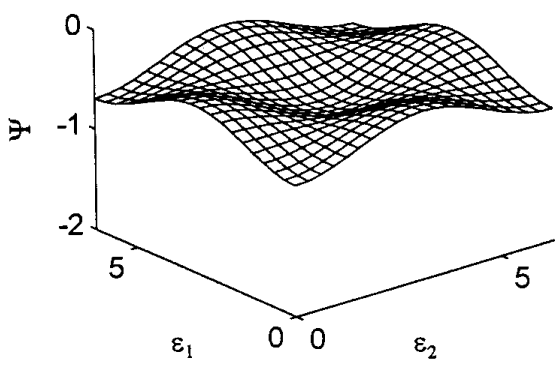

$\varepsilon_{3}=2$

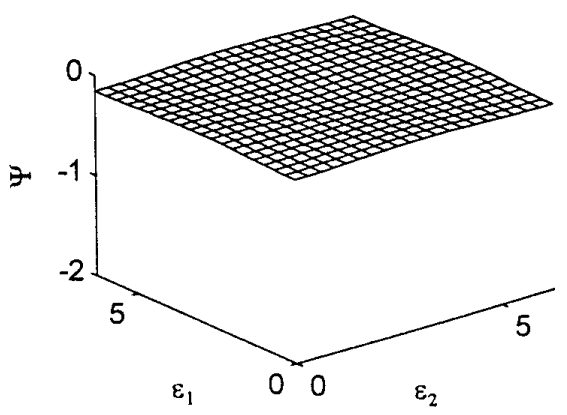

FIG . 4. Simulated spatial electrical potential distribution for the case of a nonuniformly charged rigid planar surface. The rigid surface is represented by $\epsilon_{3}=0$, and its surface potential is $g\left(\epsilon_{1}, \epsilon_{2}\right)=\cos \left(\epsilon_{1}\right) \cos \left(\epsilon_{2}\right)+1$.

As an example, consider a thin disk of radius $R$ with surface condition

$$
g\left(\epsilon_{1}, \epsilon_{2}\right)=\left\{\begin{array}{l}
f(R), \quad R \leqslant R_{m} \\
0, \quad R>R_{m}
\end{array}\right.
$$

where $R=\sqrt{\epsilon_{1}^{2}+\epsilon_{2}^{2}}$. The electrical potential distribution for $\epsilon_{3}>0$ is

$$
\begin{aligned}
\Psi\left(R, x_{3}\right) & =\frac{1}{2 \pi} \int_{0}^{R_{m}} \tilde{R} f(\tilde{R}) \int_{0}^{2 \pi} \\
& \times \frac{\exp \left(-\sqrt{\left.(R-\tilde{R} \cos \tilde{\theta})^{2}+(\tilde{R} \sin \tilde{\theta})^{2}+x_{3}^{2}\right)}\right.}{\sqrt{(R-\tilde{R} \cos \tilde{\theta})^{2}+(\tilde{R} \sin \tilde{\theta})^{2}+x_{3}^{2}}} d \tilde{R} d \tilde{\theta} .
\end{aligned}
$$

In a study of the electrical interactions between two thin, charged disks, Hsu and Tseng (20) derived the expression

$$
\begin{array}{r}
\Psi\left(R, x_{3}\right)=\int_{0}^{R_{m}} \tilde{R} f(\tilde{R}) \int_{0}^{\infty} \frac{\lambda}{\sqrt{1+\lambda^{2}}} J_{0}(\lambda \tilde{R}) J_{0}(\lambda R) \\
\times \exp \left(-\sqrt{1+\lambda^{2}} x_{3}\right) d \lambda d \tilde{R} .
\end{array}
$$

It can be shown that

$$
\begin{aligned}
& \int_{0}^{\infty} \frac{\lambda}{\sqrt{1+\lambda^{2}}} J_{0}(\lambda \tilde{R}) J_{0}(\lambda R) \exp \left(-\sqrt{1+\lambda^{2}} x_{3}\right) d \lambda \\
& \quad=\int_{0}^{2 \pi} \frac{\exp \left(-\sqrt{\left.(R-\tilde{R} \cos \tilde{\theta})^{2}+(\tilde{R} \sin \tilde{\theta})^{2}+x_{3}^{2}\right)}\right.}{2 \pi \sqrt{(R-\tilde{R} \cos \tilde{\theta})^{2}+(\tilde{R} \sin \tilde{\theta})^{2}+x_{3}^{2}}} d \tilde{\theta},
\end{aligned}
$$

and Eq. [34] is equivalent to Eq. [35].

Figure 5 shows the electrical potential distribution for the case

$$
f(R)=\left\{\begin{array}{rc}
-1, & R \leqslant 0.9 \\
2, & 0.9<R \leqslant 1 . \\
0, & 1<R
\end{array}\right.
$$

This type of surface condition simulates that of a kaolin particle in a low pH aqueous solution (21). In this case the basal plane of a particle is negatively charged and its edge positively charged (22).

\subsection{Thin, Arbitrary Surface}

For illustration, let us consider the axis-symmetric, thin charged surface formed by rotating the curve defined below about the $z$ axis: 

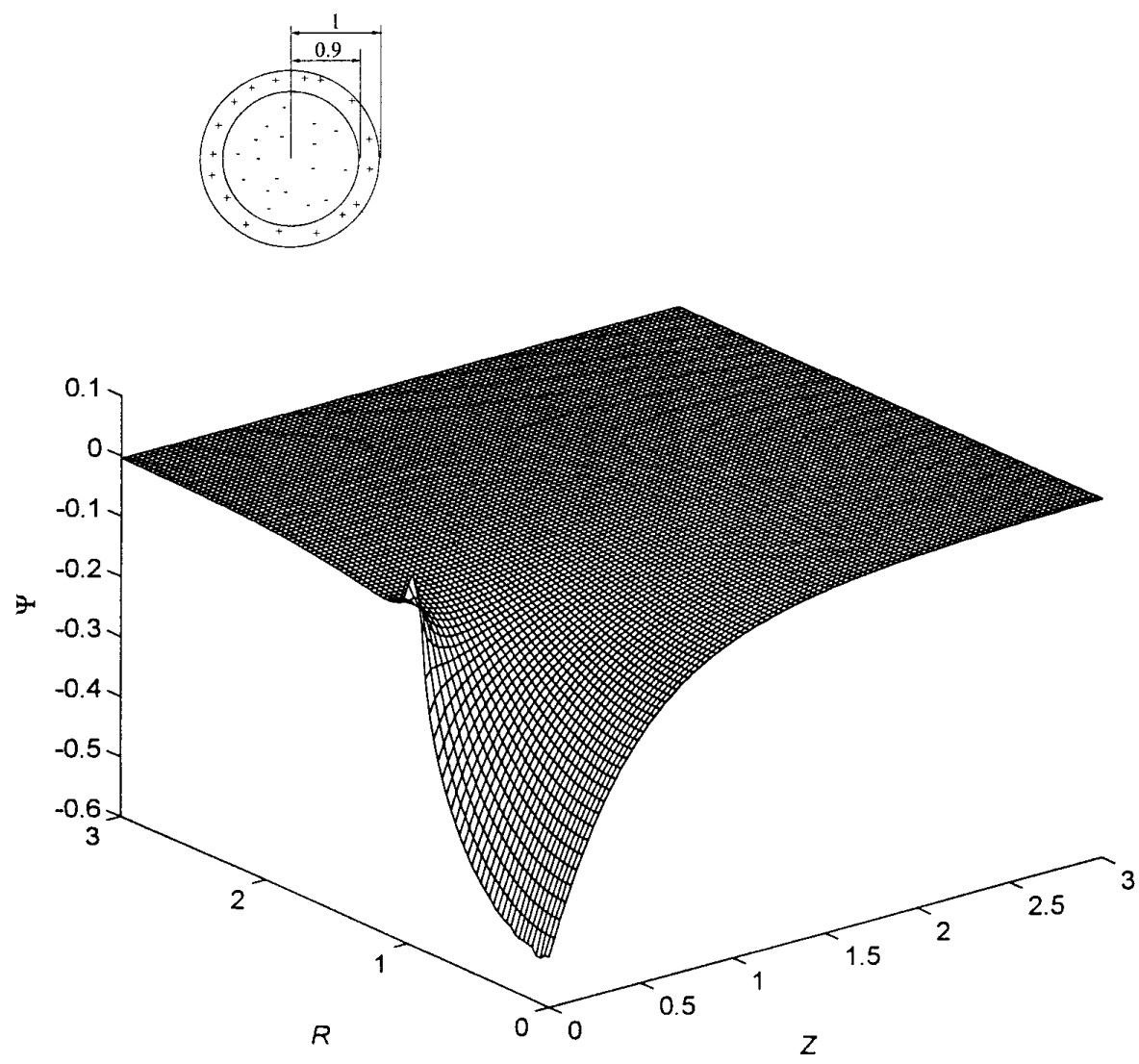

FIG. 5. Simulated electrical potential distribution over an infinitesimally thin, charged disk for the case the surface condition is defined in Eq. [37].

$$
\left\{\begin{array}{l}
r=f(t) \\
z=g(t)
\end{array}, 0 \leqslant t \leqslant t_{\max }\right.
$$

Suppose that $f(t) \geqslant 0, f(0)=f\left(t_{\max }\right)=0$, and $d g(t) / d t$ $\geqslant 0,0 \leqslant t \leqslant t_{\max }$. Under these conditions the surface defined is a simple closed surface with surface charge density $\rho_{s}(\bar{\epsilon})=\rho_{s}(t)$. The potential distribution can be calculated by Eq. [12]. We have

$$
\begin{aligned}
\Psi(r, z)= & \int_{0}^{t_{\max }} \int_{0}^{2 \pi} \exp (-R) \rho_{s}(t) \\
& \times f(t) \sqrt{\left(f^{\prime}(t)\right)^{2}+\left(g^{\prime}(t)\right)^{2}} /(4 \pi R) d \theta d t,
\end{aligned}
$$

where $R=\left((r-f(t) \cos (\theta))^{2}+(f(t) \sin (\theta))^{2}+(z-\right.$ $\left.g(t))^{2}\right)^{1 / 2}$.

Figure 6 shows the surface defined by rotating the curve

$$
\left\{\begin{array}{l}
r=t(5-t)\left(\sin ^{2}(t)+0.3\right) /(t+3) \\
z=t(t+1) / 15
\end{array}, 0 \leqslant t \leqslant 5\right.
$$

about the $z$ axis (or $\epsilon_{3}$ axis). The contours for the electrical potential distribution on the $r-z$ plane for the case $\rho_{s}=1$ are shown in Fig. 7. Since the electrical potential distribution is axis-symmetric, only those on the half $r-z$ plane are presented.

\section{CONCLUSION}

We show that the exact solution to the linearized PoissonBoltzmann equation governing the electrical potential distribution of a charged surface in an electrolyte solution under the Debye-Huckel condition can be expressed as

$$
\Psi=\int_{V} G(\bar{\epsilon}, \bar{x}) \rho(\bar{x}) d \bar{\epsilon}
$$

where $G(\bar{\epsilon}, \bar{x})$ is the generalized Green function of the linearized Poisson-Boltzmann equation. Equation [41] is valid for the case the fixed charges are distributed in the liquid phase; e.g., they are carried by an ion-penetrable membrane. If the fixed charges are distributed over a rigid surface, an image method can be applied which transforms the original problem to a form that Eq. [41] is applicable. The distribution $\rho(\bar{x})$ in both cases can be arbitrary. Most of the reported results for the linearized Poisson-Boltzmann equation in the 


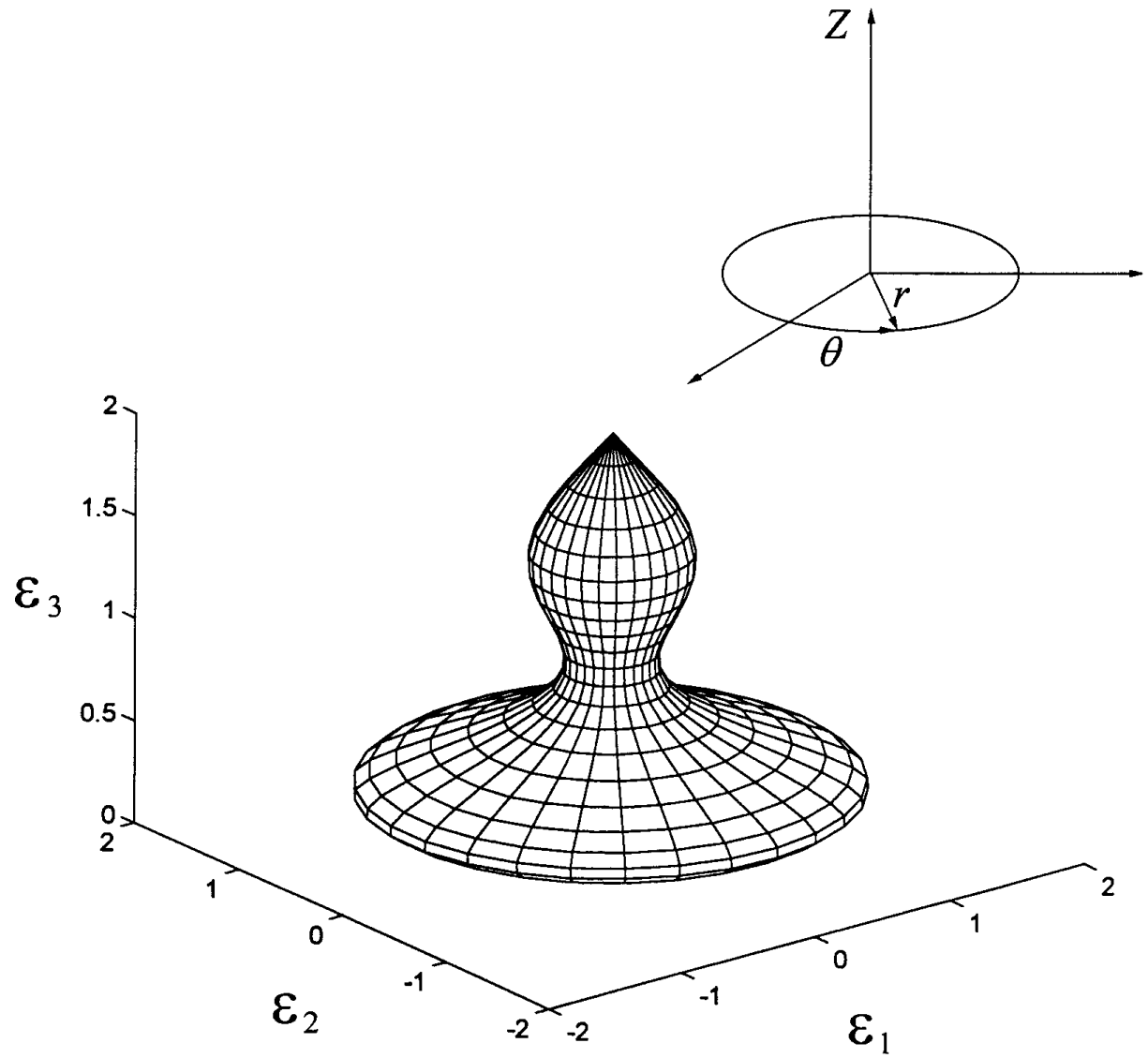

FIG. 6. An arbitrary thin surface obtained by rotating the curve defined by Eq. [40] about the $\epsilon_{3}$ axis.

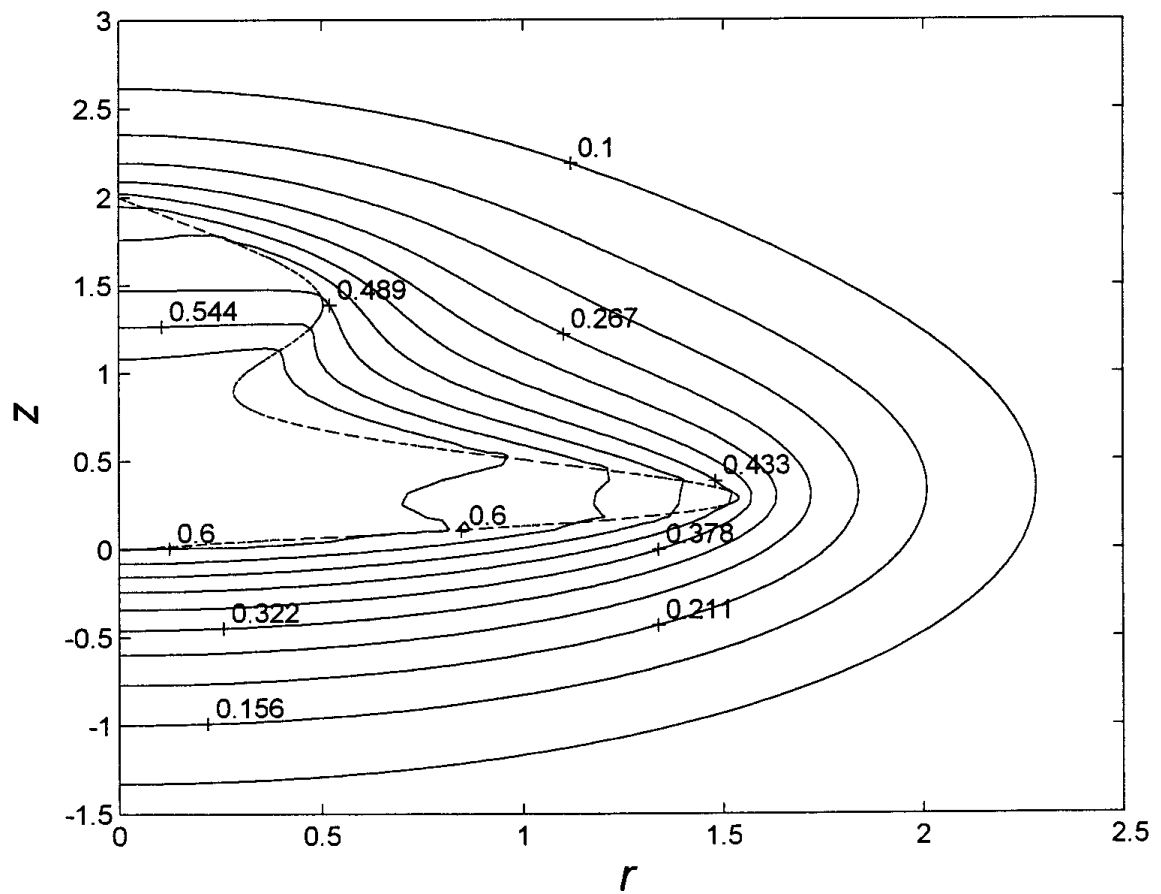

FIG. 7. The contours of the electrical potential distribution on the $r-z$ plane for the case of Fig. 6 . The surface charge density is $\rho_{s}=1$. The dashed line denotes the curve described by Eq. [40]. 
literature can be recovered as special cases of the present analysis.

\section{ACKNOWLEDGMENT}

This work is supported by the National Science Council of the Republic of China under project NSC85-2214-E002-023.

\section{REFERENCES}

1. Guenther, R. B., and Lee, J. W., "Partial Differential Equations of Mathematical Physics and Integral Equations.' Prentice Hall, London, 1982.

2. Hunter, R. J., 'FFoundations of Colloid Science,', Vol. I. Oxford Univ. Press, London, 1989.

3. Carnie, S. L., Chan, D. Y. C., and Stankovich, J., J. Colloid Interface Sci. 165, 116 (1994).

4. Chang, F. C., and Sposito, G., J. Colloid Interface Sci. 163, 19 (1994).

5. Anandarajah, A., and Chen, J., J. Colloid Interface Sci. 168, 111 (1994).

6. Parlange, J. Y., J. Chem. Phys. 57, 376 (1972).
7. Stokes, A. N., J. Chem. Phys. 65, 261 (1976).

8. Hsu, J. P., and Kuo, Y. C., J. Chem. Soc. Faraday Trans. 89, 1229 (1993).

9. van Aken, G. A., Lekkerkerker, H. N. W., Overbeek, J. Th. G., and de Bruyn, P. L., J. Phys. Chem. 94, 8468 (1990).

10. Glendinning, A. B., and Russel, W. B., J. Colloid Interface Sci. 93, 95 (1983).

11. Carnie, S. L., Chan, D. Y. C., and Gunning, J. S., Langmuir 10, 2993 (1994).

12. Ohshima, H., Adv. Colloid Interface Sci. 53, 77 (1994).

13. Kuin, A., J. Chem. Soc. Faraday Discuss. 90, 235 (1990).

14. Hsu, J. P., and Liu, B. T., J. Colloid Interface Sci. 175, 785 (1996).

15. Yoon, B. J., and Kim, S., J. Colloid Interface Sci. 128, 275 (1989).

16. Halle, B., J. Chem. Phys. 102, 7239 (1995).

17. Hsu, J. P., Hsu, W. C., and Chang, Y. I., J. Colloid Interface Sci. 165, 1 (1994).

18. Ohshima, H., and Kondo, T., Biophys. Chem. 39, 191 (1991).

19. Hsu, W. C., Hsu, J. P., and Chang, Y. I., J. Colloid Interface Sci. 155, 1 (1993).

20. Hsu, J. P., and Tseng, M. T., Langmuir, accepted.

21. Sherwood, J. D., and Stone, H. A., Phys. Fluids 7, 697 (1995).

22. Nabzar, L., Pefferkorn, E., and Varoqui, R., J. Colloid Interface Sci. 102, 380 (1984). 